\title{
ADDITIONS AND CORRECTIONS TO THE LIST OF NON-BITING MIDGES IN SASKATCHEWAN
}

\author{
P.G. MASON, Agriculture Canada Research Station, 107 Science Place, \\ Saskatoon, Saskatchewan, S7N 0X2, and D.W. PARKER, AquaTax Consulting, \\ 1204 Main Street, Saskatoon, Saskatchewan. S7H OL2
}

Since publication of the list of chironomids known to occur in Saskatchewan in "An amateur naturalist's guide to non-biting midges in Saskatchewan"1, 69 additional species have been found to occur in the province (Table 1). ${ }^{2,3,4,5}$ This brings the total number of species to 190 . Several names have been synonomized (i.e., the names were used for species thought to be new but found to be previously described). Also, a number of typographical errors appeared in the original list during typesetting and are corrected here (Table 2).

The total number of named species now stands at 169 . Twenty-one additional species have not yet been assigned a name. Many of the species listed for North America have distributions in Alberta and Manitoba, and it is probable that they also occur in Saskatchewan but have yet to be discovered. ${ }^{4}$ The 190 species make chironomids the most diverse aquatic insect group in Saskatche- wan. They also represent about $10 \%$ of the 2,000 species thought to occur in North America (the Nearctic) and about $4 \%$ of the estimated 5,000 species worldwide. ${ }^{4}$

1. MASON, P.G., D.W. PARKER and P. MORRILL. 1991. An amateur naturalist's guide to non-biting midges in Saskatchewan. Blue Jay 49:174-182.

2. DOSDALL, L.M. and P.G. MASON. 1981. A chironomid (Nanocladius (Plecopteracoluthus) branchicolus: Diptera) phoretic on a stonefly (Acroneuria lycorias: Plecoptera) in Saskatchewan. Can. Ent. 113:141147.

3. MASON, P.G. 1986. The larvae and pupae of Stictochironomus marmoreus and S. quagga (Diptera: Chironomidae). Can. Ent. 117:43-48.

4. OLIVER, D.R., M.E. DILLON and P.S. CRANSTON. 1990. A catalogue of Nearctic Chironomidae. Ag. Can. Publ. 1857/B. 89 pp.

5. PARKER, D.W. 1992. Emergence phenologies and patterns of aquatic insects inhabiting a prairie pond. Ph.D. thesis, Department of Biology, University of Saskatchewan. 173 pp.

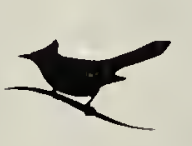

Maximum butterfly longevity: Cabbage Butterfly: 3 days for female/17 for male; tiger Swallowtail, 3/12; Spring Azure, 4/2; Orange Sulphur, 14/25; Viceroy, -/17; Great Spangled Fritillary, -/22. Paul Opler and George Krizek, Butterflies East of the Great Plains. 
Table. 1. ADDITIONS TO THE CHIRONOMIDAE KNOWN TO OCCUR IN

SASKATCHEWAN.

\section{Podonominae}

Boreochlini

Boreochlus persimilis (Johannsen)*

Lasiodiamesa rawsoni Brundin*

\section{Podonomini}

Parochlus kiefferi (Garrett) ${ }^{\star}$

\section{Tanypodinae}

Coelotanypodini

Clinotanypus (Clinotanypus) sp.

\section{Macropelopiini}

Apsectrotanypus johnsoni (Coquillett)*

Macropelopia decedens (Walker)*

\section{Pentaneurini}

Ablabesmyia (Ablabesmyia) aspera (Roback)*

Ablabesmyia (Asayia) annulata (Say)*

Conchapelopia fasciata Beck \& Beck*

Conchapelopia pallens (Coquillett)*

Guttipelopia rosenbergi Bilyj

Helopelopia cornuticaudata (Walley)*

Labrundinia pilosella (Loew)*

Larsia canadensis Bilyj ${ }^{\star}$

Meropelopia flavifrons (Johannsen) ${ }^{\star}$

Paramerina fragilis (Walley)

Zavrelimyia sinuosa (Coquillett) ${ }^{\star}$

\section{Procladiini}

Procladius (Holotanypus) culiciformis (L.)*

Procladius (Holotanypus) dentus Roback*

Procladius (Holotanypus) ruris Roback*

\section{Prodiamesinae}

Monodiamesa sp.

\section{Orthocladiinae}

Acricotopus lucens (Zetterstedt)

Brillia flavifrons (Johannsen)*

Corynoneura scutellata Winnertz*

Cricotopus (Cricotopus) cylindraceus (Kieffer \& Thiennemann)

Cricotopus (Cricotopus) flavocinctus (Kieffer)

Cricotopus (Cricotopus) tremulus (L.) ${ }^{\star}$

Cricotopus (Isocladius) pilitarsus (Zetterstedt) ${ }^{\star}$

Cricotopus brunnicans (Walley)*

Heterotrissocladius changi Saether ${ }^{\star}$

Heterotrissocladius oliveri Saether ${ }^{\star}$

Mesosmittia acutistylus Saether

Nanocladius (Nanocladius) distinctus (Malloch)*

Nanocladius (Plecopteracoluthus) branchicolus Saether

Orthocladius (Euorthocladius) rivulorum Kieffer*

Orthocladius (Orthocladius) smolandicus Brundin

Orthocladius (Symposiocladius) lignicola Kieffer

Parakiefferiella subaterrima (Malloch) ${ }^{\star}$

Parametriocnemus lundbeckii (Johannsen)*

Psectrocladius (Psectrocladius) barbimanus (Edwards)* 


\section{Chironominae}

Chironomini

Beckidia tethys (Townes)

Chironomus (Camptochironomus) pallidivittatus Malloch*

Chironomus (Chironomus) hyperboreus Staeger*

Chironomus (Chironomus) utahensis Malloch*

Cryptochironomus blarina Townes*

Dicrotendipes lucifer (Johannsen)*

Dicrotendipes tritomus (Kieffer) ${ }^{*}$

Endochironomus subtendens (Townes)*

Glyptotendipes amplus Townes*

Glyptotendipes dreisbachi Townes*

Glyptotendipes unacus Townes*

Kiefferulus (Kiefferulus) dux (Johannsen)*

Pagastiella ostansa (Webb)*

Parachironomus forceps (Townes)

Parachironomus monochromus (Wulp) *

Parachironomus varus (Goetghebuer)

Phaenopsectra punctipes (Wiederholm)*

Polypedilum (Polypedilum) nubeculosum (Meigen) ${ }^{\star}$

Sergentia (Sergentia) coracina (Zetterstedt)*

Stictochironomus marmoreus (Townes)

Stictochironomus quagga (Townes)

Stictochironomus rosenschoeldi (Zetterstedt)*

Pseudochironomini

Pseudochironomus middlekauffi Townes

Tanytarsini

Micropsectra connexa (Kieffer)*

Paratanytarsus kaszabi group

Paratanytarsus tenuis group

Stempellinella sp.

Tanytarsus buckleyi Sublette

Tanytarsus cf. mendax (Kieffer)

* from Oliver et al. (1990) ${ }^{4}$

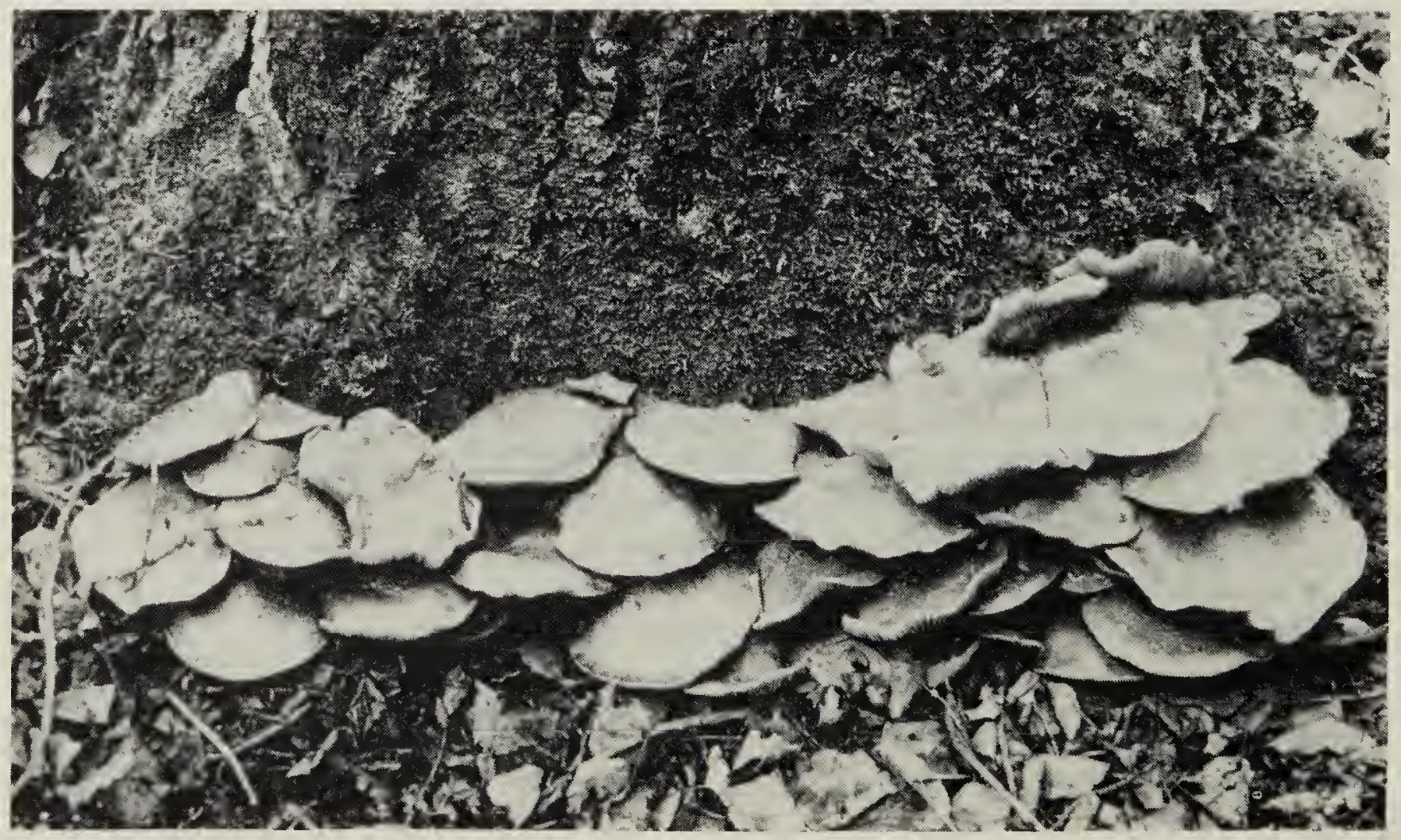


Table 2. CORRECTIONS TO THE CHIRONOMIDAE KNOWN TO OCCUR IN SASKATCHEWAN.

\section{Tanypodinae}

Macropelopiini

Derotanypus alaskensis (Malloch) was incorrectly assigned to Pentaneurini

Psectrotanypus dyari (Coquillett) was incorrectly assigned to Procladiini

\section{Pentaneurini}

Ablabesmyia (Karelia) illinoense (Malloch) should read Ablabesmyia (Karelia)

\section{Procladiini} illinoensis (Malloch)

Procladius (Procladius) denticulatus Sublette should read Procladius (Halocladius) denticulatus Sublette

Procladius (Procladius) freemani Sublette should read Procladius (Halocladius) freemani Sublette

\section{Diamesinae}

\section{Diamesini}

Diamesa cineralla Meigen should read Diamesa cf. cinerella Meigen

\section{Orthocladiinae}

Acricotopus senex (Johannsen) is now a junior synonym of Acricotopus nitidellus (Malloch)

Cricotopus (Cricotopus) bicintus (Meigen) should read Cricotopus (Cricotopus) bicinctus (Meigen)

Cricotopus (Cricotopus) curtuss Jirvenjo should read Cricotopus (Cricotopus) curtus Hirvenoja

Cricotupus (Isocladius) intersectus (Staeger) should read Cricotopus (Isocladius) intersectus (Staeger)

Cricotupus (Isocladius) ornatus (Meigen) should read Cricotopus (Isocladius) ornatus (Meigen)

Cricotupus (Isocladius) sylvestris (Fabricius) should read Cricotopus (Isocladius) sylvestris (Fabricius)

Nanocladius (Nanocladius) crassocornis Saether should read Nanocladius (Nanocladius) crassicornis Saether

Orthocladius (Orthocladius) obrumbatus Johannsen should read Orthocladius (Orthocladius) obumbatus Johannsen

Parakiefferiella (Parakiefferiella) tortulata Saether is now a junior synonym of Parakiefferiella (Parakiefferiella) subaterrima (Malloch)

Psectrocladius (Allosectrocladius) flavus (Johannsen) should read Psectrocladius (Allopsectrocladius) flavus (Johannsen)

Psectrocladius (Psectrocaldius) flavus (Johannsen) should read Psectrocladius

\section{Chironominae} (Psectrocladius) flavus (Johannsen)

\section{Chironomini}

Cladopelma viridulus (Linnaeus) should read Cladopelma viridula (Linnaeus)

Cryptochironomus stylifera Johannsen is now a junior synonym of Cryptochironomus psittacinus Meigen

Cryptotendipes casuaris (Townes) should read Cryptotendipes casuarius (Townes)

Polypedilum (Polypedilum) obtusum Townes is now a junior synonym of Polypedilum (Polypedilum) convictum (Walker)

Polypedilum (Tripodura) digitifer Townes is now a junior synonym of Polypedilum (Tripodura) halterale (Coquillett)

Stenochironomus hilarus (Walker) should read Stenochironomus hilaris (Walker) Xenochironomus (Anceus) scopulus Townes is now known as Axarius scopula

\section{Tanytarsini}

(Townes)

Micropsectra polits (Malloch) should read Micropsectra polita (Malloch)

Paratanytarsus confusus Palmen is now a junior synonym of Paratanytarsus dissimilis (Johannsen) 